\title{
Bernhard Chiari \\ Kabul, 1979: Militärische Intervention und das Scheitern der sowjetischen Dritte-Welt-Politik in Afghanistan
}

Während in der Bundesrepublik Deutschland Kinder mit ihren neuen Weihnachtsgeschenken spielten, landeten in der Nacht vom 25. auf den 26. Dezember 1979 die ersten von 7000 Elitesoldaten der sowjetischen 103. Luftlandedivision in Kabul und Bagram, etwa 80 Kilometer im Norden der afghanischen Hauptstadt gelegen. ${ }^{1}$ Sie nahmen zunächst die beiden Flughäfen und weitere Schlüsselobjekte in Kabul in Besitz. Unter dem Decknamen „Schtorm (Sturm) - 333“ erreichten Teile eines insgesamt 650 Mann starken Sonderkommandos der militärischen Aufklärung (Hauptverwaltung für Aufklärung, Glavnoe Rasvedyvatel'noje Upravlenije, GRU) und der Staatssicherheit (Komitee für Staatssicherheit, Komitet Gosudarstvennoj Bezopasnosti, KGB), am 27. Dezember den Regierungspalast „Tadsch Bek" auf einem Hügel nahe der Stadt. Spezialkräfte in afghanischen Uniformen liquidierten den afghanischen Chefkommunisten, Präsidenten und Führer der regierenden Demokratischen Volkspartei Afghanistans (DVPA), Hafisullah Amin. ${ }^{2}$ Als seinen Nachfolger setzte die sowjetische Führung Babrak Karmal ein, Gründungsmitglied der 1965 ins Leben gerufenen DVPA, der auch Amin seit ihren Anfängen angehörte. ${ }^{3}$

Kurz nach den erfolgreichen Operationen in Kabul und der Besetzung weiterer Flugfelder im Land überschritten Truppenteile der 108. Mot.-Schützendivision den Fluss Amudarja, die südliche Grenze der UdSSR zu Afghanistan. Seit November 1979 hatte das Politbüro die Einberufung von Reservisten für Verbände im Militärbezirk Turkestan (Turkestanskij voennyj okrug) verfügt und schweres Brückengerät an den Wasserlauf schaffen lassen. ${ }^{4} \mathrm{Am}$ 27. und 28. Dezember stieß die 5. Garde-Mot.-Schützendivision auf Herat vor. Motorisierte Bodentruppen nutzten die große afghanische Ringstraße als Bewegungslinie. ${ }^{5}$ Ihr Angriff bildete den Beginn der größten militärischen Operation, die sowjetische Streitkräfte sieht man von den Interventionen in Ländern des Warschauer Paktes ab - in der Nachkriegszeit außerhalb der UdSSR durchführten. Der sowjetische Vormarsch traf nur in wenigen Städten auf den Widerstand afghanischer Truppen. Ohne nennenswerte Verluste verlegte die Führung in Moskau 80000 Mann im Landmarsch sowie im Lufttransport nach Afghanistan. Ende Januar 1980 stand die Masse der sowjetischen 40. Armee im Land, bestehend aus zwei Mot.-Schützendivisionen, einer Luftlandedivision, einer Luftsturmbrigade sowie zwei selbständigen Mot.-Schützenregimentern und einem Hauptquartier im usbekischen Termes, mit Moskau direkt über Satellitenkommunikation verbunden. ${ }^{6}$

\footnotetext{
1 Nikolaj F. Ivanov, Operaciju „Štorm“ načat' ran'še, Moskau 1993, S. 315. Für die kritische Durchsicht des Manuskriptes danke ich meinen Kollegen Michael Thomae und Rüdiger Wenzke (Militärgeschichtliches Forschungsamt, MGFA) sowie Conrad Schetter (Zentrum für Entwicklungsforschung).

${ }^{2}$ Sergej P. Krachmalov, Zapiski voennogo attaše. Iran - Egipet - Iran - Afganistan, Moskau 2000, S. 220.

${ }^{3}$ Ivanov, Operaciju „Štorm“ načat' ran'še, S.316.

${ }^{4}$ Joseph J. Collins, The Soviet Invasion of Afghanistan. A Study in the Use of Force in Soviet Foreign Policy, Lexington, Mass. 1986, S. 71.

${ }^{5}$ Krachmalov, Zapiski voennogo attaše, S. 219.

${ }^{6}$ The Soviet-Afghan War. How a Superpower Fought and Lost. The Russian General Staff, übersetzt und hrsg. von Lester W. Grau und Michael A. Gress, Lawrence, Kansas 2002, S. 12; Collins, The Soviet Invasion of Afghanistan, S. 71.
} 
Die Besetzung Afghanistans folgte dem Muster des sowjetischen Einmarsches in die Tschechoslowakei 1968. Hier wie dort versuchte man, eine kommunistische Führung zweifelhafter Loyalität und Zuverlässigkeit abzusetzen. In der ČSSR wie in Afghanistan inthronisierten die Sowjets neue, Moskau genehme Regime. Zwei der wichtigsten sowjetischen Beobachter, die den Einmarsch von Kabul aus vorbereitet hatten - General Aleksej A. Epišev, Chef der Politischen Hauptverwaltung (PHV) der Sowjetarmee, und General Ivan G. Pavlovskij, stellvertretender Verteidigungsminister und Chef der sowjetischen Landstreitkräfte -, hatten eine ähnliche Funktion auch in der ČSSR erfüllt, Pavlovskij sogar als Befehlshaber der Interventionstruppen. ${ }^{7}$

Im Gegensatz zur Tschechoslowakei, wo die UdSSR 20 kriegsstarke Divisionen zum Einsatz gebracht hatte, war das sowjetische Afghanistan-Kontingent lediglich dazu in der Lage, Städte und wichtige Verkehrsverbindungen oder Nachschublinien zu sichern. Von Beginn an stand außer Zweifel, dass die eingesetzten Kräfte nicht ausreichten, um flächendeckend die afghanischen Provinzen zu überwachen. Der sowjetische Einmarsch bildete den Auftakt für einen neun Jahre währenden Krieg zwischen den sowjetischen Interventionstruppen und der Armee der neuen kommunistischen Regierung in Kabul auf der einen sowie einem Heer von Stammeskriegern auf der anderen Seite. ${ }^{8}$ Die Intervention rief zunächst einen spontanen Aufstand der afghanischen Stämme nach traditionellen Mustern und später einen Heiligen Krieg (Dschihad) hervor, den die Mudschaheddin, die islamischen Gotteskrieger, von den unzugänglichen Gebirgsregionen Afghanistans und von Pakistan aus führten. ${ }^{9}$ Dabei erhielten sie immer wirksamere materielle und logistische Unterstützung durch die Regierungen und Geheimdienste vor allem der USA und Chinas. ${ }^{10}$

Trotz erheblicher technischer Überlegenheit gelang es den Besatzern nicht, dem Aufstand im Land, der mehr und mehr die Gestalt eines Bürgerkrieges annahm, ein Ende zu setzen. Neun Jahre lang dauerte der aussichtslose Kampf gegen kaum fassbare Guerillakrieger. Bis zu 15000 sowjetische Soldaten und mehr als 1,2 Millionen Afghanen verloren dabei ihr Leben. ${ }^{11}$ Nach fünf Jahren sowjetischer Okkupation befand sich nahezu jeder zweite von insgesamt 15 Millionen Afghanen auf der Flucht. ${ }^{12}$ Erst die nach dem Amtsantritt von Generalsekretär Michail Gorbačev im März 1985 durch die Perestrojka veränderte

7 Collins, The Soviet Invasion of Afghanistan, S. 77; Pierre Metge, Die Sowjetunion in Afghanistan. Von der Zusammenarbeit zur Besetzung: 1947 bis 1986 (= Militärpolitik Dokumentation 45/46, 1985), S.50-53.

${ }^{8}$ Als Überblick zum militärischen Verlauf siehe Agha Z. Hilali, Afghanistan: The Decline of Soviet Military Strategy and Political Status, in: The Journal of Slavic Military Studies, Vol. 12 (1999), No. 1, S.94-123.

${ }^{9}$ Michael Pohly, Krieg und Widerstand in Afghanistan, Berlin 1992, S. 253-261.

${ }^{10}$ Als Überblick dazu: Afghanistan: Eight Years of Soviet Occupation, United States Department of State, Special Report No. 173, prepared by Craig Karp, Washington, D. C., December 1987, sowie die ausführliche Chronik der Besatzungszeit bei Tom Rogers, The Soviet Withdrawal from Afghanistan. Analysis and Chronology, Westport, Conn. 1992, S.61-223.

$"$ Lester W. Grau/Mohammad Nawroz, The Soviet Experience in Afghanistan, in: Military Review, 1995 (9/10), S. 17-27, hier S. 20; Odd Arne Westad, Prelude to Invasion: The Soviet Union and the Afghan Communists, 1978-1979, in: The International History Review, Vol. XVI (1994), No. 1, S. 49-69, hier S.49, spricht von bis zu 50000 getöteten Sowjets, bezieht jedoch anscheinend jene sowjetischen Soldaten mit ein, die infolge von Krankheit oder Verwundung starben. Aktuelle Berechnungen in der Russländischen Föderation, die im Internet veröffentlicht werden, gehen von 15000 gefallenen Soldaten aus (vgl. detailliert unter http://www.aeronautics.ru/nws001/afghanlosses01.htm, allerdings ohne Quellenangaben). 12 Conrad Schetter, Kleine Geschichte Afghanistans, München 2004, S. 104. Die sowjetischen Verluste beziffert Schetter auf 14000 bis 40000 , vgl. ebenda. 
geopolitische Lage und das internationale Genfer Afghanistan-Abkommen von 1988 schufen die Voraussetzungen für den Abzug der sowjetischen Truppen aus Afghanistan bis zum 14. April 1989. ${ }^{13}$ Rückzug und Niederlage hatten gravierende Auswirkungen auf das sowjetische System: Der Afghanistan-Krieg beschleunigte den Zerfall bestehender Strukturen und förderte eine Entwicklung, an deren Ende sich die UdSSR am 26. Dezember 1991 nach einem Beschluss des Obersten Sowjets auflöste. ${ }^{14}$

\section{Ideologische und außenpolitische Rahmenbedingungen}

Die sowjetische Propaganda erklärte 1979, die UdSSR habe durch die Intervention „unheilvollen Plänen zur Erdrosselung der afghanischen Revolution" durch den internationalen Imperialismus begegnen müssen, dem „das verbrecherische Handeln des Verräters Amin und seiner Clique in die Hände“ gespielt habe. ${ }^{15}$ Das Zentralkomitee der KPdSU verlautbarte am 27. Dezember, die „,eitlich befristete Aktion“ (vremennaja akcija) der sowjetischen Streitkräfte müsse die Errungenschaften der kommunistischen Machtergreifung vom April 1978 retten. ${ }^{16}$ Unmittelbar Beteiligte sahen die militärische Operation als „Export einer Revolution"17 bzw. als den Versuch, am Hindukusch unterdrückte und ausgebeutete Kleinbauern (bednjaki) zu verteidigen. ${ }^{18}$ Am 31. Dezember 1979 schrieb das OstBerliner „Neue Deutschland“: „Eine feste Faustregel sagt: Wenn Imperialisten schreien: ,Demokratie in Gefahr! Friede in Gefahr! Völkerrecht in Gefahr!‘ ist jedesmal eine Revolution vorangekommen. Heute ist es die afghanische. "19

Westliche Analysten deuteten die Intervention als „Flucht nach vorn“, nachdem die UdSSR trotz erheblichen finanziellen Engagements im Rahmen jahrelanger Entwicklungsund Wirtschaftshilfe an die Grenzen ihrer Möglichkeiten gekommen sei, in Afghanistan Einfluss auszuüben. Und tatsächlich musste die Revolution Nur Mohammed Tarakis und Amins, die im April 1978 die kommunistische Demokratische Volkspartei Afghanistans (DVPA) an die Macht brachte, zum Zeitpunkt des sowjetischen Einmarsches - aus der Perspektive des Kreml - als gescheitert, zumindest aber als nicht mehr beherrschbar gelten. ${ }^{20}$

\footnotetext{
${ }^{13}$ Hannes Adomeit/Dieter Braun, Lagenotiz betr.: Der sowjetische Rückıug aus Afghanistan. Voraussetzungen, Bedingungen und mögliche Auswirkungen des Genfer Abkommens, Stiftung Wissenschaft und Politik, SWP - LN 2566, 5/1988; ausgewählte Schlüsseldokumente in: Gorbachev and Afghanistan, hrsg. von Christian F. Ostermann, Cold War International History Project Bulletin, Issue 14/15, 2003/4, S. 143-192.

${ }^{14}$ Rafael Reuveny/Aseem Prakash, The Afghanistan War and the Breakdown of the Soviet Union, Indiana Center for Global Business, Discussion Paper No. 123, Bloomington, Ind. 1996.

15 W. F. Isgarschew, Notizen aus Afghanistan, Berlin (Ost) 1981, S. 3.

${ }^{16}$ Auszug aus dem Sitzungsprotokoll der 177. Sitzung des Politbüros, No.P177/151, 27.12.1979, Collection Soviet Invasion of Afghanistan, Cold War International History Project (CWIHP), www. CWIHP.org (Woodrow Wilson International Center for Scholars). Bezüglich der ursprünglichen Fundorte der zitierten Archivalien wird auf die dortige Dokumentation verwiesen.

${ }_{17}$ Aus der Perspektive eines ukrainischen Kriegsteilnehmers Gennadij P. Korž, Afganskoe dos'e. Istorija vojny SSSR v Afganistane, Char'kov 2003, S.3.

${ }^{18}$ Afganskaja vojna: kak eto bylo. Fotoal'bom, hrsg. von Jurij V. Platonov, Moskau 1991, S. 5.

19 Neues Deutschland, 31.12.1979, zitiert nach Paul Bucherer-Dietschi u.a. (Hrsg.), Strategischer Überfall - das Beispiel Afghanistan, I. Teilband: Das Geschehen bis Jahresende 1979, Liestal 1991, S. 254.

20 Nikolaj I. Marčuk, Neob"javlennaja vojna v Afganistane: oficial'naja versia i uroki pravdy, Moskau 1993.
} 
Unter dem Deckmantel des Sozialismus vollzog sich ein innerafghanischer Machtkampf, und hier hatte Hafisullah Amin durchaus Erfolge vorzuweisen.

Andere Beobachter nahmen die Intervention als sowjetisches Streben nach der Weltmacht wahr, als Abkehr vom Prinzip der Koexistenz hin zur Konfrontation im Äußeren. Die UdSSR habe mit dem Khyberpass das klassische Einfallstor in den Subkontinent erreicht. ${ }^{21}$ Schließlich betonte man, die Führung der UdSSR habe die Folgen ihres Handelns falsch antizipiert und sich in ihrem opportunistischen Verlangen, in Afghanistan ein entstandenes Machtvakuum zu füllen, in das Abenteuer des Einmarsches hineinziehen lassen. ${ }^{22}$ Afghanistan und "die Afghanen" spielten in den Betrachtungen überwiegend die Rolle passiver Opfer im Kampf der UdSSR und der Vereinigten Staaten um die Vorherrschaft in der Region - tatsächlich bildeten innerafghanische Entwicklungen jedoch einen wesentlichen Grund für die Intervention.

Das Zentralkomitee der UdSSR selbst informierte die Staaten des Warschauer Paktes über einen konterrevolutionären Angriff der seitens der pakistanischen Regierung unterstützten "Muslimbruderschaft“, die in großem Umfang Bewaffnete über die kaum gesicherte Grenze nach Afghanistan schleuse, um dort einen Heiligen Krieg gegen die Regierung zu führen, einen Volksaufstand zu entfachen und Akte des Terrors und der Diversion zu verüben. Eingebunden in den Aufstand seien reaktionäre Gruppen schiitischer Muslime ebenso wie eine "Clique von Maoisten“, trainiert in der Volksrepublik China. ${ }^{23}$ Umfangreiche Propagandamaßnahmen der UdSSR sollten die Aufmerksamkeit der Weltöffentlichkeit auf die Unterstützung lenken, die die Aufständischen unter anderem aus Pakistan, den USA, Iran und China erhielten. ${ }^{24}$ Aber selbst innerhalb des östlichen Bündnisses waren die Reaktionen uneinheitlich. Während Bulgarien, die ČSSR und die DDR die sowjetische Entscheidung in vollem Umfang unterstützten und tatkräftige Solidarität übten, brachten andere Staaten des Warschauer Paktes Zweifel an der Legitimation und Sinnhaftigkeit der Intervention zum Ausdruck. Ungarn hielt sich mit Kommentaren anfangs merklich zurück und vermittelte auch bei späteren Bekundungen von Zustimmung bestenfalls den Eindruck einer Pflichtübung. Noch reservierter verhielten sich die polnischen Kommunisten, die zum Jahresende 1979 nicht einmal das Glückwunschtelegramm Edward Giereks an den neuen afghanischen Staatschef Babrak Karmal veröffentlichten. Rumäniens Staats- und Parteichef Nicolae Ceauçescu schließlich kritisierte offen den sowjetischen Schritt und erhob grundsätzliche Einwände gegen das Vorgehen der UdSSR..$^{25}$

Unterschiedliche Faktoren beeinflussten die Entscheidung für den Einmarsch. Zunächst bietet für die ausgehenden 1970er Jahre der heute häufig vernachlässigte Gesichtspunkt

${ }^{21}$ Gert Linde, Des Kremls Weg zum Khỵberpass (= Berichte des BIOST, 23-1978), Köln 1978, S. 19.

22 Astrid von Borcke, Die sowjetische Interventionsentscheidung: Eine Fallstudie zum Verhältnis sowjetischer Außen- und Innenpolitik, in: Heinrich Vogel (Hrsg.), Die sowjetische Intervention in Afghanistan. Entstehung und Hintergründe einer weltpolitischen Krise, Baden-Baden 1980, S. 119-180, hier S. $119 \mathrm{f}$.

23 Information über die Ereignisse in Afghanistan für die Führung Ungarns, übermittelt durch den sowjetischen Botschafter, Vladimir Pavlov, 29.3.1979, Collection Soviet Invasion of Afghanistan, CWIHP.

24 Entscheidungen des Politbüros der KPdSU über Afghanistan, 18.3. 1979, Collection Soviet Invasion of Afghanistan, CWIHP.

25 Christian Meier, Die sowjetische Intervention in Afghanistan und die Reaktion im Warschauer Pakt, in: Vogel (Hrsg.), Die sowjetische Intervention in Afghanistan, S. 273-298. 
von Ideologie und Prestige einen wesentlichen Hintergrund. Die Rechtfertigung der Intervention fußte auf der leninistischen Revolutionstheorie in Verbindung mit der sowjetischen Militärdoktrin. Leonid Brežnev hatte den afghanischen Kommunisten am 5. Dezember 1978 „revolutionäre Solidarität“ zugesichert und versprochen, die Ergebnisse der afghanischen April-Revolution zu schützen. Die UdSSR sah sich wie alle sozialistischen Staaten verpflichtet, im Zeichen des „proletarischen Internationalismus“ national- oder sozialrevolutionäre „Volksmassen“ oder „Fortschrittskräfte“ in anderen Ländern im Kampf gegen die „äußerste Reaktion“ im Innern oder gegen fremde „Imperialisten“ zu unterstützen. Im Falle Afghanistans fand die Lehre vom „Befreiungsbürgerkrieg“ Anwendung, einem Haupttyp der sowjetischen Kriegsartenlehre. ${ }^{26}$

Die sowjetische Propaganda berief sich 1979 auf ein Hilfeersuchen der afghanischen Regierung, auf Artikel 4 des sowjetisch-afghanischen Freundschaftsvertrages vom 5.Dezember 1978 sowie - mit Blick auf eine angebliche Bedrohung der UdSSR von Pakistan aus - auf das Recht auf Selbstverteidigung nach Artikel 51 der Charta der Vereinten Nationen. Alle drei Begründungen für die Invasion hielten allerdings schon einer oberflächlichen Betrachtung nicht stand: Den einzig möglichen Urheber eines Hilfeersuchens, den ungeliebten Afisullah Amin, töteten Spezialkräfte unmittelbar nach dem Einmarsch. Die sowjetisch-afghanischen Verträge von 1921 und 1931, die das Abkommen von 1978 ergänzte, aber nicht außer Kraft setzte, schlossen eine militärische Intervention explizit aus. Die Bekämpfung von Gegnern der kommunistischen Regierung in Kabul schließlich missachtete alle Verfahren der Vereinten Nationen zur Konfliktregelung. Dies machte auch die Berufung auf die UN-Charta unglaubwürdig. Außenminister Andrei Gromyko selbst sprach im Politbüro die Konsequenzen an, die die Sowjetunion im Gegenzug zur fortdauernden Kontrolle über das rückständige, wirtschaftlich kaum entwickelte Afghanistan in Kauf nehmen müsse: „Wir müssen in Betracht ziehen, dass wir auch vom rechtlichen Standpunkt keine Rechtfertigung hätten, Truppen zu entsenden. Nach der UN-Charta kann ein Land um Unterstützung bitten, und wir könnten Truppen schicken, wenn es Ziel einer Aggression von außen ist. Afghanistan war nicht das Ziel irgendeiner Aggression. Das ist seine innere Angelegenheit, ein revolutionärer interner Konflikt, der Kampf einer Bevölkerungsgruppe gegen eine andere. Im Übrigen haben uns die Afghanen nicht offiziell gebeten, Truppen zu entsenden." Und KGB-Chef Jurij V. Andropov brachte es in der PolitbüroSitzung vom 19. März 1979 auf den Punkt: „Truppen entsenden würde heißen, Krieg gegen das Volk zu führen, das Volk zu vernichten, auf das Volk zu schießen. Wir werden aussehen wie Aggressoren, und wir werden das nicht verhindern können." ${ }^{27}$

In der Ära Brežnev wurde unter anderem die Doktrin von bewaffneten Interventionen als Mittel der Außenpolitik in der UdSSR festgeschrieben. Militärisches Eingreifen oder die Unterstützung nationaler Befreiungsbewegungen konnten sich über den direkten sowjetischen Hegemoniebereich hinaus auf alle Bereiche des Globus richten, solange dies den Interessen des „proletarischen und sozialistischen Internationalismus“ und damit der UdSSR diente. 1979 schien das marxistische, sowjetisch unterstützte Regime in Kabul zusammenzubrechen. Hierauf wird im Folgenden noch näher einzugehen sein. Von den USA und China als auch von den Dritte-Welt-Ländern wäre dies sowie die Passivität der

\footnotetext{
${ }^{26}$ Helmut Dahm, Afghanistan als Lehrstück der politischen und militärischen Doktrin Sowjetrußlands, in: Vogel (Hrsg.), Die sowjetische Intervention in Afghanistan, S. 181-246, hier S. 196-200.

27 Mitschrift des Treffens des Politbüros des ZK der KPdSU, 19.3. 1979, Collection Soviet Invasion of Afghanistan, CWIHP.
} 
Supermacht Sowjetunion als Zeichen der Schwäche verstanden worden. ${ }^{28}$ Das Ende der kommunistischen Herrschaft in Afghanistan hätte in Moskauer Augen eine schwere Niederlage des Sozialismus bedeutet und einen erheblichen Glaubwürdigkeitsverlust für dessen Führungsmacht mit sich gebracht. ${ }^{29}$

Die Entscheidungsträger in der UdSSR handelten dabei in dem Bewusstsein, dass die Zeichen insgesamt günstig standen für die Ausweitung des sowjetischen Machtbereichs. Die Niederlage der USA in Vietnam hatte diese Einschätzung ebenso gestärkt wie die sowjetischen Erfolge bei der Unterstützung linker Bewegungen in Angola und Äthiopien. Die Geiselnahme von Teheran, wo am 4 . November 1979 alle Angehörigen der US-Vertretung durch aufgebrachte Studenten festgesetzt und teils bis 20.Januar 1981 festgehalten wurden, führte der Welt die Grenzen US-amerikanischer Dominanz vor Augen. Die zweite Runde der Abrüstungsverhandlungen über strategische Waffen (SALT II) hatte am 18. Juni 1979 in Wien zur Unterzeichnung eines Abkommens geführt und die 1972 in Genf begonnenen Gespräche erfolgreich beendet. ${ }^{30}$ In der Carter-Administration waren die Befürworter einer Politik in der Mehrzahl, die die UdSSR nicht durch Konfrontation vom Verhandlungstisch fernzuhalten versuchte. ${ }^{31}$ Auch die verhaltene Reaktion der amerikanischen Regierung auf die Stationierung sowjetischer Verbände auf Kuba im September 1979 schien die Hoffnung nahezulegen, die westliche Welt werde nach einer Intervention am Hindukusch rasch wieder zur Tagesordnung übergehen. ${ }^{32}$

Gleichzeitig sah sich das Politbüro bedrohlichen globalen Entwicklungen gegenüber: Eine Annäherung Chinas an die Vereinigten Staaten, die NATO und Japan machte eine Veränderung des Kräftegleichgewichts in Asien zuungunsten der UdSSR denkbar, während die NATO am 12. Dezember 1979 die Stationierung von Mittelstreckenraketen in Europa beschloss. ${ }^{33}$ Im Iran hatte sich ein fundamentalistisches islamisches Regime etablieren können. Hier wie in Pakistan und den Ländern am Persischen Golf befanden sich die Islamisten auf dem Vormarsch. ${ }^{34}$ Auch in Afghanistan traten seit der zweiten Hälfte der 1960er Jahre radikale islamische Gruppen auf. Sie bekämpften die Versuche König Sahir Schahs und später des Regimes Dauds, das Land zu säkularisieren, standen allerdings mit ihrer Vorstellung von einer islamischen Revolution auch im Gegensatz zum traditionellen Islam. ${ }^{35}$ Nach der Niederschlagung eines Aufstandes im Pandschir-Tal flohen die Anhänger der radikalen „Muslimjugend“ 1975 nach Pakistan. Dort entstanden neue Gruppen wie die „Islamische Gemeinschaft Afghanistans“ und die „Islamische Partei Afghanistans“, Mo-

${ }^{28}$ Quadir A. Amiryar, Soviet Influence, Penetration, Domination and Invasion of Afghanistan, Ann Arbor, MI 1990, S.214-220.

${ }^{29}$ Wolfgang Berner, Der Kampf um Kabul. Lehren und Perspektiven der sowjetischen Militärintervention in Afghanistan (= Berichte des BIOST, 14-1980), Köln 1980, S. 11 .

30 Alvin Z. Rubinstein, Soviet Policy toward Turkey, Iran, and Afghanistan. The Dynamics of Influence, New York 1982, S. 170.

31 Henry S. Bradsher, Afghan Communism and Soviet Intervention, Oxford 1999, S. 75-117.

${ }^{32}$ Henning Behrens, Die Afghanistan-Intervention der UdSSR. Unabhängigkeit und Blockfreiheit oder Mongolisierung Afghanistans: Eine Herausforderung für das internationale Krisenmanagement, München 1982, S.64.

${ }^{33}$ Amiryar, Soviet Influence, S.232f.; Agha 7. Hilali, China's Response to the Soviet Invasion of Afghanistan, in: Central Asian Survey 20 (20)1), No. 3, S. 323-351.

${ }^{34}$ Information des KGB an die Außenpolitische Kommission des Zentralkomitees, 10. 10. 1979, Collection Soviet Invasion of Afghanistan, CWIHP.

35 Conrad Schetter, Kleine Geschichte Afghanistans, München 2004, S.91 f. 
toren des Widerstandes gegen die Kabuler Regierung und Ursprung zahlreicher wichtiger Führer der Mudschaheddin. ${ }^{36}$

Am 5.Juli 1977 rief in Pakistan Muhammad Zia-ul-Haq nach einem Putsch das Kriegsrecht aus. Zia unterstützte die Gegner der afghanischen Kommunisten auf vielfältige Weise, stellte in seinem Land Trainingscamps zur Verfügung und gab den Mudschaheddin die Möglichkeit, von hier aus ihre Angriffe gegen die Regierung in Kabul vorzubereiten und zu führen. Zias Bündnis aus Armee und islamischer Geistlichkeit hätte lieber heute als morgen den Zusammenbruch des prosowjetischen Regimes in Kabul gesehen. ${ }^{37}$ Vertreter von KGB und sowjetischem Innenministerium malten die Gefahr eines „Brückenkopfes“ für subversive Aktionen gegen die Sowjetunion an die Wand, würden islamische Rebellen am Hindukusch den Sieg über die Kabuler Führung erringen. ${ }^{38}$

Die US-Regierung setzte in Afghanistan keine Zeichen, die auf eine ernsthafte Reaktion im Falle einer Intervention hindeuteten. Eine solche Einschätzung stärkte die Passivität der Regierung Carter sowohl nach der Machtergreifung der afghanischen Kommunisten im April 1978 als auch nach der Ermordung des amerikanischen Botschafters Adolph Dubs am 14. Februar 1979 in Kabul: Dubs war von islamischen Regimegegnern gekidnappt worden und kam unter ungeklärten Umständen bei einem Feuergefecht mit afghanischen Sicherheitskräften ums Leben. ${ }^{39}$ Die afghanische Regierung düpierte ungestraft die westliche Supermacht. Ihr Außenminister war während der Krise für die US-Botschaft nicht zu erreichen und fehlte später sowohl unter den Kondolenzgästen der Kabuler Vertretung wie bei der Überführung von Dubs Leichnam in die Vereinigten Staaten. ${ }^{40}$ Als kurz vor dem Einmarsch am 8. und 9. Dezember 1979 die Aufklärung der Vereinigten Staaten die Verlegung eines sowjetischen Fallschirmjäger-Bataillons nach Bagram erkannte, beließ es die US-Regierung bei Nachfragen in Moskau nach den sowjetischen Absichten in Afghanistan. ${ }^{41}$

Wesentlich für den sowjetischen Entschluss war nicht die Hoffnung, Afghanistan für den Sozialismus zu gewinnen, sondern die Angst davor, mit dem Zusammenbruch des dortigen Regimes die Ergebnisse von Jahrzehnten substanziellen Engagements im Land aufs Spiel zu setzen. Die endgültige Einbindung Afghanistans in den sowjetischen Machtbereich hatte am 27. und 28. April 1978 begonnen, als die "Saur-Revolution“ die autoritäre Regierung von Mohammad Daud stürzte und Nur Mohammed Taraki als Premierminister sowie Afisullah Amin als Außenminister ihr Land zur Demokratischen Republik erklärten. Der Umbau Afghanistans startete als Putsch von Armeeoffizieren, die Daud 1973 teils selbst mit zur Macht verholfen hatten. Der neue afghanische Präsident und seine Führungsmannschaft repräsentierten die 1965 gegründete Demokratische Volkspartei Afghanistans (DVPA), die seitdem das Land der UdSSR zugewandt hatte. Die Partei konnte 1978 bereits auf eine langjährige Geschichte friedlicher Koexistenz mit der Sowjetunion und auf erhebliche Entwicklungshilfe seitens des Nachbarn zurückblicken. ${ }^{42}$

\footnotetext{
${ }^{36}$ Mahmut A. Garejew, Afghanistan nach dem Abzug der sowjetischen Truppen, Zürich 1996, S. 15.

${ }^{37}$ Collins, The Soviet Invasion of Afghanistan, S. 69.

38 Astrid von Borcke, Die Intervention in Afghanistan - Das Ende der sowjetischen Koexistenzpolitik? Determinanten des innersowjetischen Entscheidungsverhaltens (= Berichte des BIOST, 6-1980), Köln 1980 .

39 Thomas T. Hammond, Red Flag over Afghanistan. The Communist Coup, the Soviet Invasion, and the Consequences, Boulder, Col. 1984, S. 106.

40 Rubinstein, Soviet Policy toward Turkey, Iran, and Afghanistan, S. 163.

41 Metge, Die Sowjetunion in Afghanistan, S.52.

${ }^{42}$ Linde, Des Kremls Weg zum Khyberpass, S. 3-6.
} 
Die sowjetisch-afghanischen Beziehungen reichten zurück bis 1919, als die junge Russische Sozialistische Föderative Sowjetrepublik die erste sowjetische Mission nach Kabul entsandte. Am 27. März 1919 - also noch vor dem Vertrag von Rawalpindi, der im August die Unabhängigkeit Afghanistans von Großbritannien fixierte - annulierte das revolutionäre Russland sämtliche Verträge mit der afghanischen Regierung aus der Zarenzeit. In einem Brief an König Amanullah versprach Lenin sowjetische Hilfe im Falle eines Angriffs auf Afghanistan. ${ }^{43}$ Noch im Jahre 1919 besuchten sowjetische und afghanische Delegationen Kabul bzw. Moskau. ${ }^{44}$ Die sowjetische Politik der folgenden Jahre umfasste den Aufbau von Wirtschafts- und Kulturbeziehungen (Militär- und Wirtschaftsabkommen von 1921), begleitet von der aggressiven Einflussnahme auf die afghanische Innenpolitik, beispielsweise während des afghanischen Bürgerkrieges nach dem Sturz Amanullah Khans 1929. Spannungen mit der UdSSR führten in der Zwischenkriegszeit dann sogar mehrfach bis an den Rand eines militärischen Konfliktes. ${ }^{45}$ Bis in die frühen fünfziger Jahre hinein blieb Afghanistan insgesamt auf die Vereinigten Staaten hin orientiert. Dies veränderte sich erst, als die USA mit der Aufrüstung des Nachbarn Pakistan begannen. Die Rahmenbedingungen des Kalten Krieges verschafften der UdSSR die Möglichkeit einer verstärkten Präsenz am Hindukusch. ${ }^{46}$

König Daud entsetzte 1955 auf einer Loya Dschirga versammelte afghanische Stammesvertreter mit der Nachricht, dass seine Regierung die Hilfe der Sowjetunion in Anspruch nehmen werde, um das Land voranzubringen. Ein am 18. De\%ember 1955 durch Ministerpräsident Nikolaj A. Bulganin und Nikita Chruščev in Kabul unterzeichnetes Kommuniqué über die zukünftigen Wirtschaftsbeziehungen und ein wenig später von der UdSSR eingeräumter Kredit in Höhe von 100 Millionen Dollar bildeten den Beginn einer Erfolgsgeschichte sowjetischer Außen- und Entwicklungspolitik, in deren Verlauf die Sowjetunion wesentlichen Anteil am Aufbau der afghanischen Wirtschaft und Infrastruktur sowie bei der Aufstellung moderner Streitkräfte hatte. ${ }^{47} 1969$ besuchten 60000 Touristen das Land. In den 1970er Jahren schien nichts darauf hinzudeuten, dass Afghanistan innerhalb weniger Jahre im Bürgerkrieg versinken würde. ${ }^{48}$

Bis 1979 erhielt Afghanistan sowjetische Kredite in Höhe von etwa drei Milliarden Dollar. Die UdSSR ermöglichte die Realisierung bedeutender Projekte wie der 1964 fertig gestellten Salang-Straße von Kabul bis an die sowjetische Grenze und der Verbindung von Kandahar nach Herat bzw. bis zur afghanisch-sowjetischen Grenze bei Torghondi, eingeweiht 1965. In den 1970er Jahren entstanden eine Brücke über den Amudarja bei Hairatan, der Binnenhafen Schir Khan Bandar am selben Fluss oder ein Militärflugplatz bei Herat. Die Ausbeutung erheblicher Gasreserven in Afghanistan - 1973 lieferte Afghanistan 2,7 Milliarden Kubikmeter an die UdSSR - kontrollierte die Sowjetunion ebenso wie die

43 Sri Prakash Sinha, Afghanistan in Aufruhr, Freiburg u. a. 1989, S. 185.

${ }_{4}$ Afghanistan und der Nachbar im Norden, in: Vogel (Hrsg.), Die sowjetische Intervention in Afghanistan, S. 67-92, hier S. 71.

45. Sergej B. Panin, Sovetskaja Rossija i Afganistan 1919-1929, Moskau u.a. 1998, S. 14 und 196-213.

${ }^{46}$ Henry S. Bradsher, Afghanistan and the Soviet Union, neue und erweiterte Ausgabe, Durham 1985 , S. 22 .

47 Johannes Wachter, Die Krise Afghanistans 1978-1980. Ein Beitrag zur Geschichte der regionalen Ursachen der sowjetischen Interventionsentscheidung, Frankfurt a. M. u. a. 1993, S. 196-237; Agha Z. Hilali, The Soviet Penetration into Afghanistan and the Marxist Coup, in: The Journal of Slavic Military Studies, Vol. 18 (2005), No. 4, S.673-716.

48 Gilles Dorronsoro, Revolution Unending. Afghanistan: 1979 to the Present, London 2005, S. 1. 
Suche, Förderung und Nutzung von Mineralien. Die Hoffnungen sowjetischer Fachleute richteten sich auch auf die Entdeckung größerer Erdölvorkommen sowie auf die Förderung von Uran. ${ }^{49}$ Wirtschafts- und Militärhilfe gingen einher mit einer Beeinflussung des Bildungswesens und der Platzierung sowjetisch orientierter Kader an wichtigen Schaltstellen in der staatlichen Verwaltung oder in den Streitkräften. Das sowjetische Engagement mündete in einen Vertrag über die Entwicklung der wirtschaftlichen Zusammenarbeit, den beide Seiten am 14.April 1977 unterzeichneten und der auf eine Laufzeit von zwölf Jahren angelegt war. ${ }^{50}$ Insgesamt erscheint die sowjetische Afghanistanpolitik bis zur Krise von 1978/79 als langfristig angelegter, systematischer Plan zur Einbindung des Landes in die sowjetische Machtsphäre ${ }^{51}$ Der hier vorgenommene skizzenhafte Überblick macht deutlich, dass es 1979 nach sowjetischer Logik gute Gründe dafür gab, in Afghanistan den aktiven Kampf um politische, wirtschaftliche und militärische Dominanz zu führen.

\section{Revolution in der Provinz: DVPA-Kommunismus afghanischer Prägung}

Nicht der Einmarsch der Sowjetarmee 1979, sondern die Machtergreifung der Demokratischen Volkspartei Nur Mohammed Tarakis eineinhalb Jahre zuvor läutete in Afghanistan den Bürgerkrieg ein und stürzte das Land in eine schwere Krise. ${ }^{52}$ Innerhalb der Partei, seit ihrer Gründung 1965 ideell sowie finanziell abhängig von Moskau, kam es zu heftigen Machtkämpfen. Konflikte zwischen der Fraktion der "Chalk“ (Volk) unter den GhilsaiPaschtunen Taraki und Hafisullah Amin sowie den Anhängern der „Parcham“ (Banner) unter Babrak Karmal, die die DVPA seit ihrer Aufstellung erschüttert hatten, brachen erneut aus. In den folgenden Auseinandersetzungen ging es einerseits um weltanschauliche Fragen, andererseits aber auch immer um die Sicherung indivueller Machtpositionen und die Ausschaltung von Gegnern. Unter dem Deckmantel des Marxismus-Leninismus sowjetischer Prägung verfolgten die "Chalkis“ ein schillerndes Konglomerat politischer Ziele, das sozialrevolutionäre Elemente ebenso umfasste wie großafghanischen Nationalismus oder das Engagement für die Lösung der Paschtunistan-Frage, also das Streben nach einer Zusammenfassung paschtunischer Siedlungsgebiete in Afghanistan und Pakistan. Viele Führer der DVPA verstanden Ämter und Funktionen im kommunistischen Staatsapparat als Pfründe, die man an verdiente Gefolgsleute vergeben konnte. Insbesondere Hafisullah Amin bereicherte sich am Besitz König Sahir Schahs und Dauds oder verteilte diesen großzügig an eigene Günstlinge. ${ }^{53}$

49 Hans Bräker, Die sowjetische Iran- und Afghanistan-Politik, Teil I: Wirtschaftliche Beziehungen und islamisch-revolutionärer Umbruch (= Berichte des BIOST, 47-1997), Köln 1979, S. 23-26.

50 Assad Omar, Die russisch-afghanischen Beziehungen von der ersten russischen Gesandtschaft 1878/79 nach Afghanistan bis zum sowjetischen Einmarsch in Afghanistan am 27. 12. 1979, Frankfurt a. M. u.a. 1987 , S. $104 \mathrm{f}$.

51 Said Musa Samimy, Sowjetische Afghanistanpolitik. Historischer Rückblick und künftige Perspektiven, in: Uwe Halbach (Hrsg.), Afghanistan nach dem sowjetischen Truppenrückzug, BIOST Sonderveröffentlichung, Dezember 1989, S.5-27, hier S.9-12.

52 Agha Z. Hilali, The Soviet Decision-Making for Intervention in Afghanistan and Its Motives, in: The Journal of Slavic Military Studies, Vol. 16 (2003), No. 2, S. 113-145.

53 Vasili Mitrokhin, The KGB in Afghanistan, Geographical Volume 1, S. 24, Collection Soviet Invasion of Afghanistan, CWIHP. 
Taraki, Amin, Karmal und weitere Führer der DVPA verband ein komplexes, in Jahrzehnten gewachsenes Konkurrenzverhältnis, überlagert durch bestehende Stammes-, Familien- und Freundschaftsbeziehungen. Die intellektuellen Wortführer der DVAP begriffen sich dabei durchaus als Kämpfer für den Fortschritt. Die traditionelle afghanische Gesellschaftsordnung lehnten sie ebenso wie die Herrschaft lokaler Clanchefs als rückwärtsgewandt und perspektivlos ab. Das Projekt des Sozialismus sicherte in ihren Augen breiten Bevölkerungsschichten den Zugang zu Bildung und Kultur. Afghanistan öffnete sich der (sozialistischen) Welt. Erstmals verließen Menschen in größerer Zahl das Land für Zwecke der Ausbildung oder der Erholung. Mehr als 30000 Kinder zwischen sechs und 14 Jahren brachte man - teils gegen den Willen der Eltern - zum Schulbesuch in die Sowjetunion. Die neue Zeit brachte eine neue Generation Schriftsteller, Filmemacher und Künstler hervor, allerdings um den Preis der Emigration, Verhaftung oder Liquidierung jener Intellektuellen, die sich dem Sozialismus entgegenstellten. ${ }^{54}$

Die herrschende Klasse Afghanistans bestand in den 1970er Jahren aus wenigen Tausend Personen, die aufgrund von Reichtum oder Prestige über sozialen und politischen Einfluss verfügten. Zu ihnen zählten die Stammesaristokratie, Intellektuelle aus Hochschulen und Geistlichkeit, Beamte und Kaufleute. Bis 1978 übten daneben die Angehörigen der Königsfamilie, die Muhammadsai, erheblichen Einfluss in Verwaltung und Armee aus. ${ }^{55}$ Die „Chalk“ rekrutierte sich vor allem aus Studenten, Lehrern, Beamten und Journalisten. Im April 1978 umfasste die Gruppierung nicht mehr als 2000 bis 3000 Mitglieder, vor allem in Kabul. Viele ihrer Vertreter hatten vor der Machtergreifung höhere Ämter im Staatsapparat bekleidet und selbst dem afghanischen Establishment angehört.

Führer der „Chalk“ war der 1917 bei Ghazni geborene Paschtune Nur Mohammed Taraki, ein Aufsteiger aus dem Volk, dessen Karriere „Vom Schafhirten zum Revolutionsführer“ der Züricher Tages-Anzeiger belächelte. ${ }^{56}$ Nach Schule und Ausbildung arbeitete er unter anderem für eine Handelsgesellschaft in Britisch-Indien. 1937 nach Afghanistan zurückgekehrt, fand Taraki eine Anstellung im Pressebüro der Regierung in Badachschan. Taraki gehörte einer paschtunisch-dominierten Literatenbewegung an und stand als Übersetzer in Kontakt mit den USA und den Vereinten Nationen. Die Beschäftigung mit der sowjetischen Literatur brachte ihn in Verbindung mit der sowjetischen Botschaft, die den jungen Autor förderte und unterstützte. Als einer der Gründer der DVPA stand Taraki 1965 bereits in ständigem Kontakt mit dem KGB. ${ }^{57}$

Auch der zweite Mann in der Partei, Hafisullah Amin, erhielt seine Ausbildung zunächst in Kabul, wo er unterrichtete und später eine weiterführende Schule leitete. 1921 in Paghman bei Kabul geboren, studierte Amin in New York City und lebte mit seiner Familie in den USA, wo er sich intensiv mit marxistischen Ideen auseinandersetzte. Nach seiner Rückkehr nach Afghanistan arbeitete er seit 1958 in einer Ausbildungseinrichtung für Lehrer des Erziehungsministeriums in Kabul. 1962 ging er an die Columbia University und bewegte sich dort maßgeblich in marxistischen Kreisen: 1965 rief die afghanische Regierung

54 Agha Z. Hilali, The Soviet Decision-Making for Intervention in Afghanistan and Its Motives, in: The Journal of Slavic Military Studies, Vol. 16 (2003), No. 2, S. 113-145, hier S. 135.

55 Dorronsoro, Revolution Unending, S.31.

${ }^{56}$ Tages-Anzeiger (Zürich), 8.6. 1978, zitiert nach Bucherer-Dietschi u.a. (Hrsg.), Strategischer Überfall, S. 83f.

57 Vasili Mitrokhin, The KGB in Afghanistan, Geographical Volume 1, S. 1-3, Collection Soviet Invasion of Afghanistan, CWIHP; Bradsher, Afghanistan and the Soviet Union, S. 36-39. 
Amin aufgrund seiner politischen Betätigung nach Kabul zurück, wo er Mitglied der gerade gegründeten DVPA wurde..$^{58}$

Die kleinere Fraktion der „Parcham“ bekundete ebenfalls, den traditionellen Stammesund Nationalitätenpartikularismus in Afghanistan in einem säkularisierten, modernen Nationalstaat überwinden zu wollen. Parcham-Chef Babrak Karmal, 1929 bei Kabul geborener Anwalt und Sohn des Generals Muhammad Hussain Khan, Gouverneur der Provinz Paktia, war Abkomme persisch sprechender Paschtunen aus dem Kaschmir. In der Hauptstadt besuchte er die Amani-Eliteschule. Als Jurastudent verbüßte er eine mehrjährige Haftstrafe wegen marxistischer Betätigung. Karmal führte „Parcham“ im Schatten des Parteiideologen Mir Akbar Khyber, der am 17. April 1978 ermordet wurde und dessen Tod den Anlass für die kommunistische Machtergreifung bildete. 1973 hatte Karmal den Vetter des Monarchen und ehemaligen Ministerpräsidenten Mohammed Daud Khan unterstützt und beim Sturz König Sahir Schahs geholfen. „Parcham“ stellte in Dauds Republik mehrere Kabinettsmitglieder, darunter den Innenminister, bevor es 1977 zur Aufkündigung der Kooperation mit Dauds National-Revolutionärer Partei kam. Erst im August 1977 verkündeten „Parcham“ und „Chalk“ offiziell wieder die Einheit der DVPA.59

In Moskau genoss Karmal angesichts seiner intimen Beziehungen zu den afghanischen Behörden sowie zum Königshof den Ruf, einer „königlich-kommunistischen Partei“ vorzustehen. Als typisches Produkt der Kabuler Bourgeoisie stand er den afghanischen Stämmen ebenso fern wie dem bäuerlichen Leben in der Provinz. Eigene Anhänger warfen ihm vor, jenseits aller politischen Rethorik ebenso wie die "Chalk“ in Afghanistan eine Politik der paschtunischen Dominanz voranzutreiben: Anfang der 1970er Jahre spaltete sich mit „Setem-i-Meli“ (Gegen nationale Unterdrückung) ein aus Tadschiken, Usbeken und anderen Nicht-Paschtunen bestehender Kampfbund von „Parcham“ ab.

Nach der kommunistischen Machtergreifung verfolgte „Chalk“ die Anhänger der „Parcham" und schloss sie von der Arbeit in der Partei, in den Streitkräften oder im Staatsapparat aus. In vielen Fällen eskalierte der Kampf gegen die „Parchamovcy“ bis zu deren physischer Liquidierung. ${ }^{60}$ Kaum sechs Wochen nach der Revolution begannen Verhaftungen in Polizei und Armee. Am 5. Juli schied Babrak Karmal aus der Regierung aus und ging als Botschafter nach Prag. Der ebenfalls „Parcham“ nahestehende Innenminister Nur Achmad Nur sowie die Sozialministerin Anahita Ratebzad wurden auf diplomatische Posten in Washington und Belgrad abgeschoben. Im August 1978 inhaftierte die Polizei den populären Verteidigungsminister Abdul Qadir und seinen Generalstabschef Schah Pour, wenig später auch den Planungsminister Ali Kischtmand und den Minister für Öffentliche Arbeiten, Mohammed Rafi. Kabuler Zeitungen veröffentlichten Berichte über eine Verschwörung des „Aristokraten“ Babrak Karmal und seiner Anhänger. ${ }^{61}$

Taraki überzog Afghanistan mit einem Netz des Terrors. Im Land herrschten Angst und Schrecken. Im Rahmen von Massenverhaftungen säuberten die Kommunisten Behörden und Universitäten von ihren Gegnern. Die Armee übernahm dabei einen wichtigen Part und profitierte von sowjetischem Know-how. Gleichzeitig waren die Streitkräfte selbst das

\footnotetext{
58 Bradsher, Afghanistan and the Soviet Union, S. 40-43.

59 Berner, Der Kampf um Kabul, S. 22-24.

60 Vgl. die Erinnerungen des späteren Generalmajors Krachmalov, Zapiski voennogo attaše, S. 209, der als Angehöriger der sowjetischen Auslandsaufklärung sowohl die Saur-Revolution als auch den sowjetischen Einmarsch in der Botschaft Kabul erlebte.

61 Karl-Heinrich Rudersdorf, Afghanistan - eine Sowjetrepublik?, Reinbek bei Hamburg 1980, S.75-83.
} 
Ziel von Verhaftungen: Von etwa 8000 Offizieren und Unteroffizieren der afghanischen Armee wurde bis Oktober 1979 etwa die Hälfte aus politischen Gründen repressiert. Dies wiederum verstärkte die Abhängigkeit von den sowjetischen Militärberatern und Spezialisten in Afghanistan. ${ }^{62}$

Mullahs und Geistliche, Stammesführer oder Studenten, die einer regierungsfeindlichen Einstellung verdächtig waren, begrub man lebendig. Angehörigen verschwundener Beamter teilte die Polizei mit, ihre Verwandten seien in einen anderen Landesteil „versetzt“ worden. Die Gerichte verschickten Vorladungen zu angeblichen Zivil- oder Wirtschaftsverfahren. Leisteten die Betroffenen der Aufforderung Folge, verschwanden sie für immer. Vermummte Soldaten brachen nachts die Türen von Privathäusern auf und verschleppten deren Bewohner. Politische Gefangene in eilig eingerichteten Gefängnissen vegetierten in Dunkelhaft ohne sanitäre Einrichtungen dahin. Jede Verbindung zur AuBenwelt blieb ihnen versperrt. Im November 1979 veröffentlichte das Kabuler Innenministerium eine Liste mit den Namen von 12000 politischen Gefangenen, die vor der Machtergreifung Amins umgebracht worden seien. ${ }^{63}$ Ein Sinnbild für die Brutalität des Regimes stellte das Gefängnis Pule Charkhi, 25 Kilometer vor Kabul gelegen, dar, wo Tausende Häftlinge Opfer von Massenerschießungen wurden, teilweise ohne zuvor auch nur verhört zu werden. ${ }^{64}$

Der Kampf des Regimes gegen seine Gegner zeigte die Züge von Bürgerkrieg und Auflösung. ${ }^{65}$ Im Rahmen von Repressalien wurden häufig persönliche Rechnungen beglichen. ${ }^{66} \mathrm{Um}$ in den Gefängnissen Platz für die Verhafteten zu schaffen, ließ die Regierung bis Dezember 1978 nach eigenen Angaben Tausende Häftlinge frei, größtenteils gewöhnliche Straftäter und Verbrecher. Nicht einmal die Regierungsorgane verfügten über genaue Kenntnisse darüber, wen sie verhaftet und wohin gebracht hatten, wer erschossen wurde, an einer Krankheit starb oder plötzlich wieder freikam. ${ }^{67}$ Selbst viele der verbliebenen Parteimitglieder mochten unter diesen Umständen nicht mehr so recht daran glauben, dass die DVPA aus dem eigenen Verhalten einen Alleinvertretungsanspruch für das afghanische Volk ableiten könne. ${ }^{68}$ In Kabul tauchten handgeschriebene Flugblätter der „wirklichen Chalkis“ auf, die Kritik am Personenkult um Taraki und dem brutalen Führungsstil Amins äußerten.

Die UdSSR hatte am marxistischen Staatsstreich vom April 1978 nach heutigem Kenntnisstand keinen nennenswerten Anteil und betrachtete den Umsturz überaus zurückhaltend. In Moskau genoss die DVPA von Anfang an den Ruf windiger Salonkommunisten, aber offensichtlich nicht den einer ernst zu nehmenden Revolutionspartei. Die Medien der internationalen kommunistischen Bewegung schwiegen sich über den Parteichef und seine Revolution weitgehend aus. Auf der 3. Weltkonferenz der kommunistischen Parteien

62 Bradsher, Afghanistan and the Soviet Union, S. 123.

63 Arab News, Jidda (Saudi-Arabien), 18.11. 1979, zitiert nach Bucherer-Dietschi u.a. (Hrsg.), Strategischer Überfall, S. 180.

${ }^{64}$ Sinha, Afghanistan in Aufruhr, S. 51-56.

65 Sidigullah Fadai, KGB und afghanischer Widerstand, in: Ostblick: Analysen, Dokumente, Informationen, 12 (1986), S. 11-17.

66 Telegramm Außenminister Gromykos an die sowjetische Vertretung in Kabul, 15.9. 1979, Collection Soviet Invasion of Afghanistan, CWIHP.

67 Rudersdorf, Afghanistan - eine Sowjetrepublik?, S. 68-74.

ti Ergänzung zum Sitzungsprotokoll No.P149/XIV, Sitzung des Politbüros des ZK der KPdSU vom 12.4. 1979, S.4, Collection Soviet Invasion of Afghanistan, CWIHP. 
1969 fehlte die DVPA ebenso wie auf den KPdSU-Kongressen von 1966, 1971 und 1976. Auch nach der Revolution blieb Grußbotschaften der DVPA-Leitungsorgane - entgegen der in der Sowjetunion üblichen Gepflogenheiten - der Weg in sowjetische Zeitungen versperrt. ${ }^{69}$ Moskau gegenüber denunzierten sich die Vertreter von „Chalk“ und „Parcham“ gegenseitig als Spitzel und Parteigänger von CIA und Mossad, was der DVPA zusätzliche Glaubwürdigkeit in der Sowjetunion nahm. ${ }^{70}$

Im ehemaligen Königs- und Präsidentenpalast in Kabul - nun umbenannt in „Haus des Volkes“ - kündigte Taraki im Juni 1978 tiefgreifende Reformen an. Die neue afghanische Führung strebe nichts weniger als „die totale Abkehr von der Vergangenheit an, die umfassende Revolution“, und werde selbst die untersten Posten des Staatsapparates mit loyalen Anhängern der DVPA besetzen. ${ }^{71}$ Unter den Bedingungen außerhalb Kabuls weitestgehend traditionell organisierter Stammesgesellschaften erklärte die afghanische Regierung im Oktober 1978 die gesetzliche Gleichstellung von Mann und Frau. Schon die Saur-Revolution von 1978 hatten die Menschen in den Provinzen jedoch mehrheitlich als einen Kampf der Clans um die Macht verstanden. Die Herrschaft der in sich zerstrittenen und in landestypischen Verteilungskämpfen befangenen DVPA führte der sowjetischen Regierung vor Augen, dass sich eine zentral gesteuerte Umgestaltung des Landes nur unter größten Schwierigkeiten und gegen den Widerstand der Masse der ländlichen Bevölkerung erreichen ließ. Die „Diktatur des Proletariats“, so schrieben sowjetische Beobachter, müsse in Afghanistan ohne Proletariat stattfinden. ${ }^{72}$ Im Jahre 1978 waren unter 15 Millionen Afghanen kaum 20000 Industriearbeiter, im Bereich der Konstruktion nochmals etwa 50000 Menschen beschäftigt. ${ }^{73}$ Parteichef Taraki selbst erklärte dem sowjetischen Ministerpräsident Aleksej Kosygin gegenüber, dass „kaum jemand die Regierung unterstützt. In Kabul haben wir keine Arbeiter, nur Handwerker."74

Die „Chalk“ scheiterte mit dem Versuch einer rabiaten Landreform, welche die DDRFührung 1980 ihren Bürgern als wichtigen Schritt „für die Festigung der Massenbasis der neuen revolutionären Machtorgane und die Befreiung der armen Bauern und Pächter von Schuldknechtschaft und vom Würgegriff der Wucherer und Feudalherren " anpries. ${ }^{75} \mathrm{Ju}$ gendliche Aktivisten aus Schulen und Hochschulen, die die Spielregeln des Lebens in den traditionellen Stammesgesellschaften nicht einmal kannten, wurden in die Dörfer geschickt, um dort die Segnungen des Kommunismus zu verkünden. In ihrem Bestreben, unterprivilegierten Bevölkerungsgruppen Zugang zu Bildung und Boden zu verschaffen, ignorierten die afghanischen Kommunisten die Rahmenbedingungen traditioneller Stammesgesellschaften. Zwangen Repräsentanten der Regierung eine Bäuerin in eine Grund-

${ }^{69}$ Die sowjetische Invasion in Afghanistan, hrsg. von ICA/Botschaft der USA in Bonn (= Amerikadienst Dokumentation), 16.4.1980, S. 1 .

70 Berner, Der Kampf um Kabul, S. 24-29 und 31-36.

71 Andreas Kohlschlüter, Die neue Klasse Kabuls. Interview mit Nur Mohammad Taraki, in: Die ZEIT, 9.6. 1978, S.4, zitiert nach Günter Schröder (Hrsg.), Afghanistan zwischen Marx und Mohammed. Materialien zur afghanischen Revolution, Teil I, Gießen 1980, S. 111-116.

72 Aleksandr A. Ljachovskij/Vjačeslav M. Zabrodin, Tainy afganskoj vojny, Moskau 1991, S. 17-19.

${ }^{73}$ Bradsher, Afghan Communism and Soviet Intervention, S.9.

${ }^{74}$ Mitschrift des Treffens des Politbüros des ZK der KPdSU, 17.3. 1979, Collection Soviet Invasion of Afghanistan, CWIHP.

75 Revolutionäres Afghanistan, hrsg. von der Akademie der Gesellschaftswissenschaften beim Zentralkomitee der SED durch ein Autorenkollektiv unter Hans-Joachim Radde und Egon Dummer, Berlin (Ost) 1980, S. 21. 
schule, entehrten sie damit deren Ehemann: In den Dörfern setzten sich die Männer gegen eine solche Vergewaltigung herrschender Verhältnisse zur Wehr. ${ }^{76}$

Bei der Neuverteilung des Bodens legte die DVPA eine primitive Vorstellung von „Feudalherrschaft" zugrunde, die die komplexen wirtschaftlichen und gesellschaftlichen Bedingungen im Land ignorierte. ${ }^{77}$ Höchst unterschiedliche naturräumliche Gegebenheiten in den verschiedenen Provinzen vernachlässigten die Reformer ebenso wie gravierende lokale Unterschiede bezüglich der Bewässerungssysteme, der Siedlungsformen und Betriebsgrößen. Hatten traditionell die Khane, Maleks bzw. die Geistlichkeit als Mittelsmänner die Beziehungen zwischen Stadt und Land geregelt, sollten nun nicht vorhandene Behörden deren Funktionen übernehmen. Die DVPA unterschied nicht zwischen unterschiedlichen Formen des Grundbesitzes (etwa Stammes- und Dorfeigentum an Grund und Boden), sondern verteilte Ackerland über sechs Hektar unabhängig von dessen Ertrag in stets gleichen Parzellen. Neben die Unsinnigkeit der Reform traten gravierende Mängel in ihrer Durchführung. Statt wie geplant 817000 landlose Bauernfamilien auszustatten, erhielten tatsächlich bloß 210000 Familien Land zugewiesen. Missbrauch und Bestechung bei der Verteilung konnten nicht eingedämmt werden, verlässliche kartografische Unterlagen fehlten. Die Ausstattung mit Saatgut unterblieb, zwangsweise gegründete Genossenschaften konnten die Rolle der als rückschrittlich gebranndmarkten dörflichen Solidargemeinschaften nicht auffangen. Während nur ein kleiner Teil der Landbevölkerung von den Veränderungen profitierte, erlitt der Staat erhebliche Steuereinbußen. Eine Versorgungskrise machte sich bemerkbar, während die Fläche des bearbeiteten Bodens durch die Flucht von Einzelpersonen oder geschlossenen Familienverbänden immer weiter abnahm. ${ }^{78}$

Während die Regierung in Kabul versuchte, das Land mit Gewalt nach sozialistischem Muster umzustrukturieren, litt die afghanische Verwaltung zunehmend unter den Folgen der Säuberungen. In vielen Bereichen fehlte durch die Verhaftung von Regimegegnern und „Feinden“ qualifiziertes Fachpersonal. Statt die Gesellschaft zu verändern, vergrößerte die DVPA den Abstand zu den Provinzen und rief Feindschaft und Widerstand gegen die Reformbestrebungen hervor. ${ }^{79}$ Selbst in der Hauptstadt der DDR sprach man von „bestimmte[n] Überspitzungen bei der Durchführung der Generallinie der DVPA, insbesondere hinsichtlich des Tempos der Bodenreform " ${ }^{80}$ Sowjetische Diplomaten empfahlen den afghanischen Kommunisten, bei der Sowjetisierung einen behutsamen Kurs einzuschlagen, auf die regionalen und lokalen Machtverhältnisse Rücksicht zu nehmen und selbst der Religionsausübung zunächst keine Hindernisse zu bereiten. Die DVPA müsse klarmachen, dass sie Religionsführer nicht als solche verfolge, sondern lediglich gegen einzelne Extremisten vorgehe, die sich durch ihr Handeln gegen den revolutionären Aufbau stellten. ${ }^{81}$

Die Politik der DVAP rief den Widerstand breiter Bevölkerungsschichten und bewaffnete Aufstände islamischer Gotteskrieger (Mudschaheddin) hervor. Die Ablehnung des

\footnotetext{
76 Said M. Samimy, Hintergründe der sowjetischen Invasion in Afghanistan. Berichte und Analysen, Bochum 1981, S. 48 .

77 Vgl. im Wortlaut Bodenreformgeset der Demokratischen Republik Afghanistan. November 1978, in: Wolfram Brönner, Afghanistan. Revolution und Konterrevolution, Frankfurt a. M. 1980, S. $214-221$.

${ }^{78}$ Samimy, Hintergründe der sowjetischen Invasion in Afghanistan, S. 12-19 und 48.

79 Bradsher, Afghanistan and the Soviet L'nion, S.90f.

80 Revolutionäres Afghanistan, hrsg. von der Akademie der Gesellschaftswissenschaften, S. 49.

81 Ergänzung zum Sitzungsprotokoll No.P149/XIV, Sitzıng des Politbüros des ZK der KPdSU vom 12.4. 1979, S.9, Collection Soviet Invasion of Afghanistan, CWIHP.
} 
volksdemokratischen Kurses vereinte die Gegner der „Chalk“ über Stammesgrenzen hinweg. Die Lage in Afghanistan offenbarte auf dramatische Weise ein Aufstand, der am 15. März 1979 in Herat losbrach. Bewohner der Stadt, Bauern der umliegenden Dörfer und Soldaten der örtlichen Garnison lieferten sich in Herat vier Tage lang blutige Gefechte mit afghanischen Regierungstruppen. Die Märzunruhen forderten 5000 Tote, darunter 150 bis 200 sowjetische Militärberater und ihre Familienangehörigen. ${ }^{82}$ Die Aufständischen vernichteten Panzer der Taraki ergebenen afghanischen Truppen und Jeeps der sowjetischen KGB-Leute mitsamt ihren Insassen. ${ }^{83}$ Anders als bei früheren, rasch niedergeschlagenen Meutereien in Masar-i Scharif und Kabul konnten die Aufständischen Herat eine Woche lang halten. Ein überregionaler Plan oder entsprechende Netzwerke, die eine landesweite Koordinierung der Aufstände ermöglicht hätten, fehlten hingegen. Die Rebellion offenbarte den spontanen Volkszorn in den afghanischen Provinzen. Er war aber gerade darum viel erschreckender als vereinzelte Attentate der bis zu diesem Zeitpunkt noch kaum organisierten Islamisten, die 1978 nur geringen Rückhalt in der Bevölkerung fanden. ${ }^{84}$

Im sowjetischen Politbüro stieg durch den Aufstand in Herat die ohnehin vorhandene Nervosität. ${ }^{85}$ Außenminister Gromyko konnte am 17. März ebenso wenig ein klares Lagebild zeichnen wie die militärische Führung. Gravierende Zweifel herrschten hinsichtlich der Zuverlässigkeit der afghanischen Streitkräfte - etwa 100000 Mann, ausgebildet und ausgerüstet mit sowjetischer Hilfe. Das Politbüro musste zur Kenntnis nehmen, dass die 17. Division der afghanischen Armee, die im Raum Herat stationiert war, zum Teil zu den Aufständischen übergelaufen war. Der sowjetische Generalstab drängte darauf, die afghanischen Streitkräfte im Kampf gegen die „Bandenbildung“ (bandformirovanie) zu unterstützen. ${ }^{86}$ Der Aufstand in Herat könne der Anfang vom Ende der DVAP-Herrschaft im Lande sein.

Die Gefechte in Herat bildeten den Auftakt für schwere Kämpfe, in deren Verlauf im März und April Teile der Provinzen Kunar, Paktia, Badgis, Nangahar, Balch und selbst der Stadt Kabul zeitweise an aufständische Milizen verloren gingen. ${ }^{87}$ Die Bewohner der südöstlichen Provinz Nuristan verteidigten ihre traditionelle Lebensweise gegen staatliche Eingriffe, vertrieben Repräsentanten der Regierung, fackelten Polizeistationen ab und plünderten die Distriktverwaltungen. Der Staat schickte Panzer, ließ die Dörfer bombardieren und trieb deren Bewohner über die Grenze nach Pakistan. In Badachschan eskalierte die Gewalt in ähnlicher Weise, als Abgesandte der kommunistischen Regierung in brachialer Weise einen „Alphabetisierungsfeldzug“ starteten, in dessen Verlauf 1979 angeblich 5000 professionelle Lehrer und 20000 freiwillige Helfer zum Einsatz kamen. ${ }^{88}$ Im Sommer

82 Dorronsoro, Revolution Unending, S.97-102; Antony Hyman, Afghanistan under Soviet Domination, 1964-83, London 1982, S. 101 .

${ }^{83}$ Vgl. den Augenzeugenbericht Der Hut-Aufstand von Herat - ein Wendepunkt, in: Schröder (Hrsg.), Afghanistan zwischen Marx und Mohammed, Teil II, S. 47-58.

84 Pohly, Krieg und Widerstand in Afghanistan, S. 126-134.

${ }^{85}$ Mitschrift des Treffens des Politbüros des ZK der KPdSU, 17.3. 1979, Collection Soviet Invasion of Afghanistan, CWIHP.

86 Vgl. Afganistan. Spezoperacija v Kabule 1979 g. Vospominanija učastnikov. Russkie kommandos, hrsg. vom MOO „Vympel“ - Veterany podrazdelenij specnaznačenija Organov Gosbezopasnosti, Moskau 1999, S. 28-60.

87 Koř̌, Afganskoe dos'e, S. 551.

88 Wolfram Brönner, Afghanistan. Revolution und Konterrevolution, Frankfurt a. M. 1980, S.65. 
1979 tobten Aufstände in Urusgan und Dschelalabad, wo die beiden Provinzgouverneure und Hunderte kommunistischer Lehrer, Richter und Beamte ums Leben kamen. ${ }^{89}$

Fehler im Umgang mit den regierungsfeindlichen Kräften im Land schob die Moskauer Führung mehrheitlich Tarakis Stellvertreter Amin in die Schuhe. Sie machte diesen - dem überdies Verbindungen zum amerikanischen Geheimdienst, zur Regierung Pakistans und zu islamistischen Führern nachgesagt wurden - auch für schwere Exzesse gegenüber der eigenen Bevölkerung verantwortlich. ${ }^{90}$ Aber anstatt dass, wie von Moskau aus gewünscht, die Regierung Taraki durch die Ausschaltung Amins stabilisiert worden wäre, übernahm dieser am 14. September 1979 selbst die Macht, bildete die Regierung um und setzte Taraki ab. Taraki wurde - kurz nach seiner Rückkehr von einem Moskau-Besuch - nach einer Schießerei im Kabuler „Volkshaus“ von Gefolgsleuten Amins verhaftet und später in seiner Zelle erdrosselt. ${ }^{91}$ Die Zeitungen teilten an versteckter Stelle mit, er sei an einer Krankheit verstorben und sogleich beerdigt worden. Wollte die UdSSR den Zusammenbruch des prosowjetischen Regimes in Kabul verhindern, musste sie von nun an den ungeliebten Amin unterstützen, der in Moskau schon zum Zeitpunkt seiner Machtergreifung allen Kredit verspielt hatte. ${ }^{92}$

Amin bekundete in einer ersten programmatischen Rede nach seinem September-Coup mit Blick auf den beseitigten Konkurrenten Taraki, eine „Ein-Mann-Herrschaft“ werde es in Afghanistan nie mehr geben. Er selbst etablierte allerdings im Lande ein autoritäres Regime, das neben ihm keinen zweiten starken Mann duldete. ${ }^{93}$ Mit Fortdauer der Aufstände in Afghanistan erschien deren erfolgreiche Bekämpfung mit Hilfe der DVAP immer mehr in weite Ferne zu rücken. Diplomaten in Kabul sprachen offen über Amins Probleme mit Moskau und seine Suche nach möglichen neuen Partnern, während der KGB bereits über afghanische Auslandskommunisten Verbindung mit Babrak Karmal aufnahm und mit ihm über die Absetzung des Regimes in Kabul verhandelte. ${ }^{94}$ Wenige Tage vor dem Einmarsch meldete im Dezember 1979 die sowjetische Botschaft, die afghanische Bevölkerung mache zunehmend den „Großen Bruder“ im Norden für Amins Repressalien im Land verantwortlich. ${ }^{95} \mathrm{Im}$ Kreml stieg der Druck auf das Politbüro, die Krise in Afghanistan endlich in den Griff zu bekommen.

\section{Die Entscheidung zur Intervention}

Der sowjetische Machtapparat entwickelte einzigartige Mechanismen und Verfahren politischer Entscheidungsfindung. Bürokratie, Zensur und Zentralismus behinderten die Zusammenführung und Auswertung von Informationen auf mittlerer Ebene und überforder-

\footnotetext{
${ }^{89}$ Nasir Shansab, Soviet Expansion in the Third World. Afghanistan: A Case Study, Silver Spring, MD 1986 , S.5lf. und $65 \mathrm{f}$.

90) Zusammenfassend vgl. Bericht Andropors, Gromykos, Ustinovs und Ponomarevs zur Lage in Afghanistan, 31. 12. 1979, Collection Soviet Invasion of Afghanistan, CWIHP.

${ }_{91}$ Dorronsoro, Revolution Unending, S.90; Rudersdorf, Afghanistan - eine Sowjetrepublik?, S. $79 \mathrm{f}$.

92 Bericht Gromykos, Ustinovs und Tsviguns an das Politbüro, 15.9. und 29. 10. 1979, Collection Soviet Invasion of Afghanistan, CWIHP.

${ }_{93}$ Als Überblick dazu: Bradsher, Afghan Communism and Soviet Intervention, S. XI-XVIII.

${ }^{94}$ Pesönliches Memorandum Andropors an Brežnev, Collection Soviet Invasion of Afghanistan, CWIHP.

9: Meldung des 1. Botschaftssekretärs Mišin vom 16.12.1979, vgl. Ivanov, Operaciju „Štorm“ načat' ran'še, S. 302 .
} 
ten so die höchsten Instanzen. Zu den wesentlichen Akteuren wie dem Politbüro des Zentralkomitees der KPdSU, dem Zentralkomitee sowie der militärischen Führung bzw. der Leitung der Geheimdienste kamen weitere Führungszirkel wie die außenpolitische Kommission des Politbüros. Das sowjetische Außenministerium, formal Teil des Staatsapparates, unterstand faktisch unmittelbar dem Politbüro und damit der Partei. Bezüglich der Afghanistan-Krise 1978/79 stellt sich die Frage, inwiefern die dramatischen Ereignisse im Land auf diesen Apparat zurückwirkten und wie die Eigenlogik des Politbüros die Entscheidungsfindung beeinflusste, ja ob vielleicht die Diskussion um das „richtige“ Vorgehen in Afghanistan teilweise nur die Bühne für Machtkämpfe innerhalb der Partei bildete. Die Entscheidung für die Intervention wäre dann nicht als strategisches Kalkül, sondern lediglich vor dem Hintergrund kurz- und mittelfristiger Motive verständlich. ${ }^{96}$

Die Kommunistische Partei hatte 1979 die Stellung Leonid Brežnevs gestärkt, gleichzeitig spekulierten die Mitglieder des Politbüros angesichts dessen schwacher Gesundheit bereits über einen möglichen Nachfolger. Innerhalb des engsten Führungszirkels herrschten Spannungen zwischen zivilen Mitgliedern und Militärs. Letztere setzten „Sicherheit“ weitgehend mit militärischer Macht gleich und gingen von der Möglichkeit begrenzter lokaler Kriege unter dem Schirm des strategischen nuklearen Patts mit den USA aus. ${ }^{97}$ Die Vertreter des sowjetischen Rüstungskomplexes und die Führung der Streikräfte selbst hofften auf eine Aufstockung des Verteidigungsetats und wünschten grundsätzlich jeden Anlass herbei, um die vitale Bedeutung eines schlagkräftigen Militärapparates unter Beweis stellen zu können. Die Ansprüche der Streitkräfte bei der Verteilung politischer und ökonomischer Ressourcen waren für die Mitglieder des Politbüros selbstverständlicher Teil des politischen Systems. ${ }^{98}$

Berater des Politbüros reisten 1979 wiederholt nach Afghanistan, um sich dort ein Bild der Lage zu machen. Im April führte General Aleksej A. Epišev, Chef der Politischen Hauptverwaltung, ein Beraterteam nach Kabul. Im Mai entsandte Moskau Vasilij Safrančuk an die Kabuler Botschaft, um dort nach Lösungsmöglichkeiten für den laufenden Konflikt zwischen den DVPA-Fraktionen zu suchen. Mitte August folgte General Ivan Pavlovskij, stellvertretender Verteidigungsminister und Chef der sowjetischen Landstreitkräfte, der bis Oktober in Afghanistan blieb und dem Politbüro empfahl, zur Stabilisierung des Landes zunächst Hafisullah Amin die Macht zu entziehen. ${ }^{99}$ Drei Tage nach der Sitzung des Politbüros am 26. November 1979, auf dem die Entscheidung für die Eliminierung Amins fiel, traf schließlich Viktor S. Paputin in Kabul ein, erster Stellvertreter des Innenministers und zuständig für Polizei und Sicherheitsfragen. Zu diesem Zeitpunkt befanden sich bereits zwischen 3500 und 4000 Angehörige von Armee und KGB im Land. ${ }^{100}$ Während Paputins offizielle Mission in der Unterstützung der afghanischen Regierung bei der Bekämpfung Aufständischer bestand, bereitete er in Wahrheit das Ende Amins vor: Am 19. Dezember schoss ein Attentäter auf Amins Neffen Assadullah, Chef der afghanischen Geheimpolizei, und verwundete ihn tödlich. Amin selbst entkam verletzt einem Anschlag auf sein Leben. ${ }^{101}$

96 Vgl. hierzu Rubinstein, Soviet Policy toward Turkey, Iran, and Afghanistan, S. VII-X.

97 Von Borcke, Die Intervention in Afghanistan; dies., Die sowjetische Interventionsentscheidung, in: Vogel (Hrsg.), Die sowjetische Intervention in Afghanistan, S. 119-180, hier S. 130-134.

98 Amiryar, Soviet Influence, S. 239f.

${ }^{99}$ Bradsher, Afghanistan and the Soviet Union, S. 152; Bericht Ustinovs an das Zentralkomitee,

5.11.1979, Collection Soviet Invasion of Afghanistan, CWIHP.

1(6) Rubinstein, Soviet Policy toward Turkey, Iran, and Afghanistan, S. 167.

101 Amiryar, Soviet Influence, S. 228-231 und 234. 
Insgesamt rieten die Militärs mit Blick auf eine mögliche Intervention zur Vorsicht: Es waren am Ende Leonid Brežnev und Politbüro-Mitglieder wie der KGB-Chef Andropov, die sich über derartige Bedenken hinwegsetzten. Als der Chef des sowjetischen Generalstabs, Marschall Nikolaj V. Ogarkov, am 10. Dezember eindringlich davor warnte, eine Invasion in Afghanistan würde dem Islamismus in der gesamten Region Auftrieb geben, schnitt ihm Andropov das Wort ab. Er schnauzte Ogarkov an, er solle sich auf militärische Angelegenheiten konzentrieren und die Politik Leonid Brežnev und der Partei überlassen. ${ }^{102}$

Noch zum Zeitpunkt der Kämpfe in Herat verfügte das Politbüro des ZK der KPdSU über mehrere Handlungsoptionen. Hätte man, erstens, die dortige kommunistische Regierung aufgegeben, hätte sich die UdSSR aus Afghanistan zurückziehen können. Die Unterstützung des Regimes konnte zweitens einhergehen mit der Forderung, die DVPA müsse sich im Land um einen Ausgleich bemühen, um so die Opposition zu spalten. Verhandlungen unter sowjetischer Moderation schienen ebenso denkbar wie die militärische Bekämpfung der Aufständischen in den Provinzen, vornehmlich durch Artillerie und Luftwaffe. Schließlich konnte die sowjetische Führung drittens einen Regimewechsel inszenieren und danach verstärkt auf die Neuordnung Afghanistans hinwirken. Der Einmarsch und die Übernahme der Macht im Land schien angesichts solcher Alternativen gefährlich und zunächst wenig erfolgversprechend. ${ }^{103}$ Die Diskussionen im Politbüro machten deutlich, wie weit dessen Mitglieder zu Beginn der Krise davon entfernt waren, Bodentruppen nach Afghanistan zu entsenden. KGB-Chef Andropov erklärte während der Sitzung vom 17. März 1979, Panzer und Schützenpanzer könnten in Afghanistan "gar nichts retten“. ${ }^{104}$ Bei der Analyse des Aufstandes von Herat und der Taktik der Mudschaheddin fiel immer wieder der Ausdruck „Partisanenkrieg“ (partizanskaja vojna), seit dem Zweiten Weltkrieg in der Sowjetunion ein beinahe mythischer Begriff. Höhere Funktionäre in Partei und Armee, die meist selbst der Kriegsgeneration angehörten, waren sich also durchaus darüber im Klaren, welche Probleme die Ausweitung der militärischen Operationen gegen die afghanischen Aufständischen mit sich bringen mussten. ${ }^{105}$

Das Politbüro unterstützte die Regierung in Kabul mit umfangreichen Nahrungsmittellieferungen, durch die Gewährung eines höheren Preises für Erdgaslieferungen aus Afghanistan und durch die Entsendung von Spezialisten aus Armee und KGB. ${ }^{106}$ Ein Freundschaftsvertrag sah im Dezember 1978 umfangreiche Ausbildungs- und Rüstungshilfe für die afghanischen Streitkräfte vor. Der Luftwaffenstützpunkt in Bagram wurde zur logistischen Drehscheibe für sowjetische Militärtechnik, der bald Spezialpersonal, Ausbilder, Militärberater und Angehörige der Geheimdienste folgten. Am 20. März 1979 sagte Kosygin während eines persönlichen Treffens mit Taraki in Moskau umfangreiche kostenlose Rüstungshilfe zu, darunter zahlreiche Gefechts- und Luftfahrzeuge. ${ }^{107}$ Mit der Exportversion

\footnotetext{
102 Aufzeichnungen von A. A. Ljachovskij über eine Sitzung des Zentralkomitees am 10.12.1979, Collection Soviet Invasion of Afghanistan, CWIHP.

${ }^{103}$ Rubinstein, Soviet Policy toward Turkey, Iran, and Afghanistan, S. $164 \mathrm{f}$.

104 Mitschrift des Treffens des Politbüros des ZK der KPdSU, 17.3. 1979, Collection Soviet Invasion of Afghanistan, CWIHP.

${ }^{105}$ Krachmalov, Zapiski voennogo attaše, S. 215. Vgl. auch die Schrift „Taktik des Partisanenkampfes“ aus dem Iran, zitiert nach Koř̆, Afganskoe dos'e, S. 470-491, welche der sowjetischen Aufklärung für die Einschätzung der Taktik der Mudschaheddin diente.

${ }^{106}$ Gesprächsnotiz. A. M. Puzanov - N. M. Taraki, 22.3.1979, Collection Soviet Invasion of Afghanistan, CWIHP.

10733 Schützenpanzer BMP-1, 21 Hubschrauber der Typen MI-25 und MI-8Г sowie 50 schwimmfähige Radpanzer BTR-60PB und 2:5 Aufklärungsfahrzeuge sowie Fliegerabwehr-Systeme, darunter das leis-
} 
des Kampfhubschraubers Mi-24 (NATO-Bezeichnung „Hind“) erhielten die afghanischen Streitkräfte auch solche Waffensysteme, die den letzten Stand sowjetischer Militärtechnik widerspiegelten. Die schwer bewaffneten „helicopter gunships“, von ihren sowjetischen Crews als „fliegende Panzer“ (letajuščij tank) bezeichnet, wurden nach dem Dezember 1979 zum Sinnbild für den sowjetischen Herrschaftsanspruch in Afghanistan. Die dortige Kriegführung revolutionierten sie ebenso wie der Einsatz moderner MiG-21-Kampfflugzeuge, die Napalm gegen Dörfer zum Einsatz brachten, in denen man Mudschaheddin vermutete. ${ }^{108}$ Den Einsatz sowjetischer Kampfpiloten, wie von der afghanischen Regierung gefordert, lehnte das Politbüro hingegen ab und erteilte der unmittelbaren Teilnahme sowjetischer Militäreinheiten im Kampf gegen die Aufständischen wiederholt eine nachdrückliche Absage: Parteichef Taraki hatte dem sowjetischen Premierminister Kosygin in einem Telefonat am 17. März vorgeschlagen, Soldaten der Sowjetarmee mit afghanischen Uniformen sowie sowjetische Panzer und Flugzeuge mit afghanischen Hoheitsabzeichen zu versehen und gegen die Aufständischen einzusetzen. Die Sowjetunion solle „Usbeken, Tadschiken und Turkmenen in Zivilkleidung“ nach Herat entsenden, um dort für den Kommunismus zu kämpfen. Auf die Anmerkung Kosygins, die UdSSR hätte doch Hunderte afghanischer Offiziere ausgebildet, die nun als loyale Gefolgsleute der Regierung bereitstünden, entgegnete Taraki, es handele sich größtenteils um „Moslem-Reaktionäre“, auf die man sich nicht verlassen könne. ${ }^{109}$

Die Entscheidung für eine Intervention fiel zwischen März und November 1979. Taraki brachte Hilfeersuchen der DVPA-Führung wiederholt persönlich in Moskau vor, andere Bitten um Rüstungsgüter und Personal erreichten das Politbüro über die sowjetische Auslandsvertretung und den Residenten des KGB in Kabul. Zwischen dem 17. März und dem 17. Dezember 1979 wurde die afghanische Regierung insgesamt 21-mal in Moskau vorstellig und forderte Verlegung und Einsatz von bis zu zwei Divisionen (19. Juli), um gegen die Aufständischen im Land vorgehen zu können. ${ }^{110}$ Die sowjetische Führung verlegte schließlich am 8. Juli ein Bataillon der 105. Luftlandedivision von Usbekistan auf den Luftwaffenstützpunkt Bagram. Die informelle Entscheidung zur Truppenentsendung im größeren Umfang fiel wahrscheinlich Ende Oktober, also etwa zwei Monate nach der Machtübernahme Amins. Am 6. Dezember beschlossen Brežnev, Andropov, Gromyko, Dmitrij F. Ustinov und Michail A. Suslov, ZK-Sekretär, Chefideologe und Nummer zwei der Partei, 500 Mann einer Spezialabteilung der GRU nach Afghanistan zu schicken. Vier Tage später befahl Ustinov dem Generalstab die Bereitstellung einer Luftlandedivision. Die endgültige Entscheidung für den Einmarsch trafen Brežnev, Geheimdienstchef Andropov, Außenminister

tungsstarke System „Strela“ (Pfeil). Aufzeichnung des Gesprächs der ZK-Führung der KPdSU mit N. M. Taraki, 20.3.1979, zitiert nach Pierre Allan u.a. (Hrsg.), Sowjetische Geheimdokumente zum Afghanistankrieg (1978-1991), Zürich 1995, S. 27-56.

108 Bradsher, Afghanistan and the Soviet Union, S. 102.

109 Telefongespräch zwischen Aleksej Kosygin und Nur Mohammed Taraki, 17.3. 1979, Foreign Broadcast Information Service, FBIS-SOV-92-138 (17. 7. 1992), S. 30 f. (Auszüge); Boris Gromov, Ograničennyj kontingent, Moskau 1994; beides in Collection Soviet Invasion of Afghanistan, CWIHP. Auszug aus dem Sitzungsprotokoll des Politbüros des ZK der KPdSU (150/93) zu Frage der Hilfe sowjetischer Kampfhelikopter der Regierung der DRA [Demokratische Republik Afghanistan], 21.4. 1979, zitiert nach Allan u.a. (Hrsg.), Sowjetische Geheimdokumente, S. 102-105; Auszug aus dem Sitzungsprotokoll des Politbüros des ZK der KPdSU (152/159): Gewährung zusätzlicher Militärhilfe an die DRA, 24.5. 1979, zitiert nach ebenda, S. 106-112.

110 Boris Ponomarev, Berichte aus Kabul, 19.7.1979, Collection Soviet Invasion of Afghanistan, CWIHP. 
Gromyko und Verteidigungsminister Ustinov am 12. Dezember, wobei sie weder die übrigen Mitglieder des Politbüros oder das Präsidium des Obersten Sowjets noch die zahlreich vorhandenen Spezialisten befragten, die sich im Vorfeld zur Lage im Land geäußert hatten. ${ }^{11}$ Einen Tag später begannen mit der Einrichtung einer „operativen Gruppe“ im Verteidigungsministerium die unmittelbaren Vorbereitungen für die Invasion. Den Befehl zum Einmarsch erteilte Ustinov am 24. Dezember. ${ }^{112}$

Abschließend stellt sich die Frage, welches Bild die sowjetische Führung über die Lage in Afghanistan hatte. Dessen Entwicklung im 20. Jahrhundert war bestimmt durch ein kompliziertes Beziehungsgeflecht zwischen Hauptstadt und Provinzen, Stadt und Land sowie zwischen Ethnien und Stämmen. Die afghanische Gesellschaft der 1970er Jahre zerfiel entlang unterschiedlicher Trennlinien. Geht man von der sprachlichen Zugehörigkeit aus, so bildeten im Jahre 1978 Paschtunen (6,5 Millionen), Tadschiken (3,5 Millionen), Usbeken (eine Million), Hasara (870000), Aimak (800000), Turkmenen (125000), Brahui (200000), Belutschen (100000) und Nuristaner (100000) die wichtigsten Gruppen. ${ }^{113}$ Die Stammeszugehörigkeit bot ein weiteres, wesentliches Kriterium für die Selbstverortung. Außerhalb von Dorf- oder Familienverbänden machten unklare Gruppenbildungen mit teils erheblichen Überschneidungen, Mehrfachzugehörigkeiten und Loyalitäten die Durchsetzung zentraler Entscheidungen schwer. Auf dem Land regelten Patron-Klienten-Verhältnisse und uralte Stammesgesetze den Alltag und schützten die Gemeinschaft gegen Eingriffe von außen. ${ }^{114}$ Machtwechsel im fernen Kabul begriffen die Menschen in den Provinzen als Auseinandersetzungen, von denen man sich am besten fernhielt. ${ }^{115}$ Eine überwiegend illiterate, immobile „Inward-Looking Society“ hatte über Jahrhunderte erfolgreiche Mechanismen entwickelt, um sich der staatlichen Einflussnahme zu entziehen. ${ }^{116}$

Es greift zu kurz, der sowjetischen Führung zu unterstellen, sie habe 1979 im Größenwahn, ohne sorgfältige Analyse und lediglich „auf der Basis von Instinkten“ gehandelt, ausgebildet in langen Jahren gewaltsamer Expansion. ${ }^{117}$ Die Entscheidung zur Invasion fiel in einer Phase der sowjetischen Geschichte, in der die nationalen Republiken und Kulturen ebenso wie Religionen und religiöse Bewegungen verhältnismäßig viel Autonomie genossen. ${ }^{118}$ Das Politbüro war sich über die Verfasstheit der afghanischen Gesellschaft im Klaren. ${ }^{119}$ Jenseits sozialistischer Worthülsen und offizieller Kommuniqués begriff es Af-

11 Helmut Hubel, Das Ende des Kalten Krieges im Orient. Die USA, die Sowjetunion und die Konflikte in Afghanistan, am Golf und im Nahen Osten. Auswirkungen für Europa und Deutschland, München 1995, S. 136.

112 Manfred Sapper, Die Auswirkungen des Afghanistan-Krieges auf die Sowjetgesellschaft. Eine Studie zum Legitimitätsverlust des Militärischen in der Perestrojka, Münster u.a. 1994, S.61-79, Übersicht S. 68 .

113 Die in der Literatur veröffentlichten Bevölkerungszahlen schwanken mangels Erhebungsgrundlagen sowie aufgrund der Verschiedenheit der angelegten Zuordnungskritierien stark; die Angaben hier nach Louis Dupree, Afghanistan, Princeton, N. J. 1973, S.57-65; vgl. auch Dorronsoro, Revolution Unending, S. 15; ähnliche Zahlen bei Hyman, Afghanistan under Soviet Domination, S. 11.

114 Dorronsoro, Revolution Unending, S. 10.

115 Olivier Roy, The Lessons of the Soviet/Afghan War (= IISS, ADELPHI Paper 259), London 1991, S. $6 f$.

${ }^{116}$ Dupree, Afghanistan, S. 248f.

117 Bradsher, Afghan Communism and Soviet Intervention, S. 75.

118 Vgl. Boris Dubin, Gesellschaft der Angepassten. Die Brežnev-Ära und ihre Aktualität, in: Osteuropa 57 (2007), H. 12, S. 65-78, hier S.68.

119 Vgl. ausgewählte Schlüsseldokumente in: From Hesitation to Intervention: Soviet Decisions on Afghanistan, 1979, Cold War International History Project Bulletin, Issue 4, 1994, S. 70-76. 
ghanistan als ein Land, in dem Auseinandersetzungen der Stämme, die Unabhängigkeitsbestrebungen etwa der Paschtunen und der geografisch wie mental große Abstand zwischen Hauptstadt und Provinzen den sowjetischen Vorstellungen vom Fortschritt enge Grenzen setzten. ${ }^{120}$ Der KGB lieferte über viele Jahre lang erstklassige Aufklärungsberichte und warb wichtige afghanische Führer als Agenten an. ${ }^{121}$ Zuletzt im Oktober 1979 entsandte der Geheimdienst Aufklärungsteams nach Afghanistan, die dort die Stimmungslage der Bevölkerung ergründen sollten. Bezüglich einer möglichen sowjetischen Militäroperation erhielt die Führung in Moskau eine überdeutliche Einschätzung: Sowjetische Truppen in Afghanistan, so die Aufklärungsberichte, bedeuteten einen Krieg, der nicht gewonnen werden könne, ohne die gesamte afghanische Nation auszulöschen. ${ }^{122}$

Bereits im April 1979 stellte das Politbüro fest, die Kräfte der Reaktion könnten sich in Afghanistan auf eine nahezu vollständig analphabetische Gesellschaft stützen, zudem profitierten sie von schweren nationalen Auseinandersetzungen und Stammeskonflikten sowie von religiösem Fanatismus und extremem Nationalismus. Die überwiegende Masse der Bevölkerung sei weit davon entfernt, den sozialistischen Aufbau des Landes als Vorteil zu empfinden und schätze nicht dessen fortschrittlichen Charakter. Der DVPA - nach wie vor alles andere als eine Massenpartei - sei es nicht gelungen, in den Provinz-, Stadt- und Dorfverwaltungen Fuß zu fassen, um so den Umbau des Landes auch außerhalb Kabuls voranzubringen. ${ }^{123}$ Der Kampf verfeindeter Gruppierungen innerhalb der DVPA habe dazu geführt, dass die Führer wichtiger administrativer Einheiten immer wieder ausgetauscht worden seien. Taraki und Amin hätten ihre Gegner auf der oberen und mittleren Führungsebene der Parcham-Bewegung und in den Streitkräften ermorden lassen und sich damit gegen das eigene Volk gewandt. ${ }^{124}$ „Fehler in der Kaderpolitik“ (ošibki v kadrovoj politike), so Kosygin, würden der afghanischen Führung teuer zu stehen kommen. Die Sowjetunion habe dies während der Stalinzeit erfahren müssen. ${ }^{125}$ Die Regierung in Kabul erreiche weder die Geistlichkeit noch die untereinander verfeindeten Stammesführer. Der Aufbau Afghanistans brauche „Zeit und ein etappenweise planendes und gut durchdachtes Vorgehen “. ${ }^{126}$

Sowohl Andrej P. Kirilenko, Sekretär des Zentralkomitees, als auch Außenminister Gromyko stellten fest, dass der Märzaufstand in Herat nicht nur von Islamisten aus Pakistan, unterstützt aus dem Ausland, geführt werde, sondern breite Bevölkerungsschichten unter dem traditionellen Banner des Islam vereinige. Die sowjetische Armee müsse sich darauf einstellen, im Falle der Intervention auf die Bevölkerung zu schießen - mit unabsehbaren

120 Vgl. für die Provinzen Bamian, Badachschan, Kunar, Paktia und Paktika entsprechende Aussagen bei Krachmalov, Zapiski voennogo attaše, S. 214.

121 Vgl. Vasili Mitrokhin, The KGB in Afghanistan, Geographical Volume 1, Collection Soviet Invasion of Afghanistan, CWIHP. Mitrochin, selbst Angehöriger des KGB, lief 1992 nach Großbritannien über. Seine Aufzeichnungen vermitteln ein detailliertes Bild über das Engagement des sowjetischen Geheimdienstes in Afghanistan.

122 Vgl. Bradsher, Afghan Communism and Soviet Intervention, S. 77.

123 Shansab, Soviet Expansion in the Third World, S.58.

124 Ergänzung zum Sitzungsprotokoll No. P149/XIV, Sitzung des Politbüros des ZK der KPdSU vom 12.4. 1979, S.5 und 9, Collection Soviet Invasion of Afghanistan, CWIHP.

${ }^{125}$ Aufzeichnung des Gesprächs der ZK-Führung der KPdSU mit N. M. Taraki, 20.3. 1979, zitiert nach Allan u.a. (Hrsg.), Sowjetische Geheimdokumente, S. 27-56, hier S. 52.

126 Ergänzung zum Sitzungsprotokoll No. P149/XIV, Sitzung des Politbüros des ZK der KPdSU vom 12. 4. 1979, S. 1 und 3, Collection Soviet Invasion of Afghanistan, CWIHP. 
Konsequenzen für die Wahrnehmung der UdSSR in der Welt. ${ }^{127}$ Diese Erkenntnisse spiegeln auch sowjetische Versuche wider, mäßigend auf Taraki und Amin einzuwirken und die beiden Konkurrenten im Sinne der Revolution zur Versöhnung zu bewegen. ${ }^{128}$

Realistische Lagebeurteilungen änderten nichts daran, dass die Sowjetunion Schritt für Schritt in einen Krieg hineingeriet. Die Entscheidungsträger in Moskau waren insgesamt nicht dazu im Stande, aus den ihnen vorliegenden Berichten für ihr eigenes Handeln zu folgern. Am 20. März 1979, also bereits nach Ausbruch des Aufstandes in Herat, empfing Leonid Brežnev Nur Mohammed Taraki in Moskau. Der 72-jährige Brežnev empfahl die Bildung einer nationalen Front, um in Afghanistan die sozialistische Ordnung wiederherzustellen. Diese sollte die bereits bestehenden sozialen und politischen Organisationen zusammenfassen und unterstützt werden von „Arbeitern, Bauern, der kleinen und mittleren Bourgeoisie, von Intelligenzija und Studenten, der Jugend und progressiven Frauen“. Die Landbevölkerung müsse durch die Schaffung von „Armutskommissionen“ (komitety bednoty) gewonnen werden, in denen Landlose, Kleinbauern und Schuldner den Widerstand gegen Feudalherren und Gutsbesitzer (pomeščky-kapitalisty) zu organisieren hätten. Ziel der Bewegung müsse es sein, „nationale patriotische Kräfte gegen lokale und fremde Reaktionäre“ in Stellung zu bringen. Der Wirklichkeitsferne der Aufforderung Brežnevs, einer existenziellen Krise des volksdemokratischen Regimes mit den im afghanischen Kontext untauglichen Mitteln der gesellschaftlichen Mobilisierung zu begegnen, entspricht die Nüchternheit der Antwort Tarakis. In Gegenwart Kosygins, Gromykos, Ustinovs und des ZK-Sekretärs für Internationale Angelegenheiten Boris N. Ponomarev entgegnete er Brežnev, die DVPA könne das soziopolitische Leben in Afghanistan nicht durchdringen, da das Land ökonomisch rückständig und ein erheblicher Teil seiner Bevölkerung nicht ausreichend politisch entwickelt sei. ${ }^{129}$

Brežnev sagte am 20. September 1979 im Politbüro, die Ereignisse in Kabul hätten sich derart rasch entwickelt, dass es in Moskau im Grunde keine Möglichkeit gegeben habe, mit Blick auf die Krise innerhalb der DVPA und im ganzen Land Einfluss zu nehmen. ${ }^{130}$ Die Führung der UdSSR wischte Ende des Jahres alle Bedenken beiseite und entschloss sich zum Einmarsch in Afghanistan. Sie hoffte darauf, das Land mit Hilfe einer neuen kommunistischen Führung unter Babrak Karmal zu stabilisieren, um dann das eigene militärische Engagement rasch verringern zu können. ${ }^{131}$ Diese Hoffnung erwies sich bald als ebenso irrig wie der Versuch, den ausufernden Konflikt in Afghanistan mit Gewalt zugunsten der Kabuler Regierung zu entscheiden. Der Besatzung und dem Abzug der Sowjets mehr als neun Jahre später folgte der Zerfall der UdSSR. Die Intervention schuf 1979 die Rahmenbedingungen für die Fortsetzung und Radikalisierung des Bürgerkrieges, statt ihn zu beenden. Einer der Hauptgewinner der folgenden, furchtbaren Auseinandersetzungen war der internationale islamische Fundamentalismus, der heute eine weltweite Bedrohung der Sicherheit darstellt.

\footnotetext{
127 Gromyko, Andropov, Ustinov und Ponomarev an das Zentralkomitee der KPdSU, 28.6. 1979, Collection Soviet Invasion of Afghanistan, C.WIHP.

128 Dorronsoro, Revolution Unending, S.91; Entscheidungen des Politbüros zu Afghanistan, 13.9. 1979, Collection Soviet Invasion of Afghanistan, CWIHP.

129 Gesprächsmitschrift L. I. Brežnev - N. M. Taraki, 20.3. 1979, Collection Soviet Invasion of Afghanistan, CWIHP.

${ }^{130}$ Breĭnev vor dem Politbüro, 20.9. 1979, Collection Soviet Invasion of Afghanistan, CWIHP.

131 Bericht Andropovs über Gespräche mit der neuen afghanischen Führung, 5.2.1980, Collection Soviet Invasion of Afghanistan, CWIHP.
} 